\title{
LA MADRE DE LAS OBRAS COMPLETAS, DE LOS FRAGMENTOS PÓSTUMOS Y DE LA CORRESPONDENCIA DE NIETZSCHE: EL TRABAJO DE GIORGIO COLLI Y MAZZINO MONTINARI
}

The mother of Nietzsche's Complete Works, Posthumous Fragments and Collected Letters: the work of Giorgio Colli and Mazzino Montinari

\author{
Maria Cristina Fornari \\ Universidad de Lecce -Italia
}

Resumen: Giorgio Colli y Mazzino Montinari han dado vida, en los años sesenta a una de las mayores empresas culturales del siglo: la edición crítica de las Obras y del Epistolario de Friedrich Nietzsche, que se puede considerar con el título: «la madre de todas las ediciones». Sus características son, entre otras: la relectura integral de los manuscritos, su disposición en orden cronológico, el descubrimiento de las falsificaciones y omisiones, la valoración de los fragmentos póstumos. El artículo recorre brevemente las etapas de esta importante operación cultural, restituyendo un Nietzsche libre de mistificaciones y precomprensiones ideológicas.

Palabras clave: Nietzsche - edición crítica - Colli/Montinari

Abstract: Giorgio Colli and Mazzino Montinari have given life, in the 60s, to one of the greatest cultural enterprises of the century: the critical edition of Friedrich Nietzsche's Works and Epistolary, which could be considered under the title: «the mother of all editions». Its characteristics are among others: the integral rereading of the manuscripts, their arrangement in chronological order, the detection of falsifications and omissions, and the assessment of posthumous fragments. The article briefly goes through the stages of this important cultural operation, restoring a Nietzsche free of mystifications and ideological preconceptions.

Key Words: Nietzsche - Critical Edition - Colli/Montinari

1. En los años cincuenta del siglo pasado, Mazzino Montinari, un brillante investigador italiano políticamente comprometido con el partido comunista y crecido culturalmente a la sombra de la prestigiosa Escuela Normal de Pisa ${ }^{1}$, se preguntaba ante las grandes desilusiones de la política si no sería más bien la cultura «la fuerza crítica que escava y trabaja en secreto, y rompe y lleva a la descomposición las formas del Estado y de la religión que se consideran depositarias de una validez absoluta», descubriendo a Nietzsche ${ }^{2}$. Del mismo modo, aquel que había sido su joven profesor en el liceo en los años anteriores a la guerra, el filósofo Giorgio Colli, de

1. Para una biografía sintética véase la G. Campioni, «Mazzino Montinari»: voz del Dizionario Biografico degli Italiani Treccani, vol. 76 (2012).

2. «La lectura de Nietzsche, mediada por Thomas Mann, es el culmen de una serie de reflexiones que impelen a Montinari a delimitar el ámbito de la política refutando la politización total, característica en aquellos años, de muchos intelectuales». En 1956 la represión del levantamiento húngaro causó en el estudioso una fuerte crisis, que culminó en el rechazo de todo totalitarismo y en un renovado 
tradición antifascista y democrática, estaba reflexionando sobre cómo atribuir al filósofo alemán la tarea de revivificar la cultura, a través de aquella que definía «la acción Nietzsche» y que concebía como un efecto directo, inmediato, en cierta medida antihistórico, de la entelequia Nietzsche sobre la vida individual.

Al compromiso filosófico y académico —-desde 1949 era profesor de historia de la filosofía antigua en la Universidad de Pisa- Colli unía una fecunda actividad de traductor y de editor ${ }^{3}$, concibiéndola como medio para difundir temas y autores que la cultura oficial y universitaria tendía voluntariamente a ocultar y a olvidar: no extraña, por tanto, que al editor Einaudi de Turín, con quien colaboraba desde hacía algunos años, Colli le hubiese propuesto desde 1945 una nueva traducción completa de las obras del filósofo alemán, incómodo, «maldito» e impopular para una buena parte del ambiente cultural italiano, sobre todo de la izquierda.

El contrato con Einaudi para la traducción de las Opere complete de Nietzsche se firmó en 1959, pero poco tiempo después el reconocimiento en Weimar de Mazzino Montinari estaba destinado a hacerse cargo de las cartas. Como cuenta Luciano Foà, colaborador de Einaudi, que había acogido la idea de Colli con entusiasmo:

El plan de la traducción de Nietzsche había sido puesto en marcha por Colli, Einaudi y yo. Las obras habrían de ser publicadas en la serie Millenni. En 1961, Colli llegó a Turín y explicó que la visita de Montinari a Weimar, donde se conservan las cartas de Nietzsche, había mostrado la necesidad de una edición completamente reconstruida desde sus manuscritos, muchos de los cuales eran inéditos. El trabajo se amontonaba y con ello aumentaba su dimensión cultural, pero también el compromiso financiero y político. Einaudi se desentendió del proyecto y se produjo la ruptura con Colli. En julio de 1961 yo dejé Einaudi. Supe después que al poco tiempo había habido una discusión en un «miércoles» einaudiano, que concluyó con la decisión de no emprender la traducción de las obras que ya estaba programada. Conseguimos recuperar los derechos de traducción ${ }^{4}$. Un año y medio después de la aparición del primer libro Adelphi salió, en 1964, el primer volumen de las obras de Nietzsche $e^{5}$.

La elección de Einaudi revela los temores político-sociales que el nombre de Nietzsche suscitaba en la época; el consejo editorial, compuesto en su mayor parte por marxistas y socialistas liberales, había expresado fuertes reservas ideológicas sobre un autor cuyas opera omnia, todavía comprometidas con los regímenes totalitarios de derechas, habrían causado un notable embarazo. Como precisó Colli en 1961, Nietzsche formaba siempre parte del catálogo de las «monstruosida-

interés por la esfera de la cultura» (P. D’Iorio, «Introduzione» a Mazzino Montinari. L'arte di leggere Nietzsche, Firenze: Ponte alle Grazie, 1992, p. 7).

3. Desde 1958 a 1965 dirige para el editor Paolo Boringhieri de Turín la Enciclopedia di autori classici: un conjunto de noventa títulos concebidos expresamente para aquel proyecto, publicados en siete años junto con un grupo restringido de colaboradores, entre ellos Mazzino Montinari.

4. Luciano Foà se marchó de Einaudi para fundar en junio de 1962, junto a Roberto Olivetti, la casa editorial Adelphi.

5. Citado en G. Campioni, Leggere Nietzsche. Alle origini dell'edizione critica Colli-Montinari. Con lettere e testi inediti, Pisa: ETS, 1992, p. 62. 
des», y de ningún modo habría podido ser repuesto «sobre el mismo estante del Gramsci de Einaudi». Fue entonces la recién nacida editorial Adelphi quien apoyó el proyecto, con tenacidad y no sin dificultades comprensibles; y mientras se busca sin éxito un editor alemán para la publicación de la edición original, ve orgullosamente la luz en 1964 en Italia el primer volumen de la edición crítica Colli-Montinari de las Opere de Nietzsche: la «madre» de todas las ediciones ${ }^{6}$.

2. En abril de 1961 Giorgio Colli envía a su antiguo alumno y amigo a que investigase en Weimar, en Turingia. Debía tratarse de un mero y breve reconocimiento del Goethe-und-Schiller-Archiv para echar un vistazo a sus manuscritos: simplemente por ser escrupuloso, antes de abordar la traducción al italiano de la que era la edición acreditada y en circulación de los escritos nietzscheanos ${ }^{7}$. Montinari partió con el entusiasmo propio de la juventud y de la militancia: en la entonces República Democrática Alemana, con todas las dificultades y desconfianzas que caracterizaban la adormecida pero alarmada Alemania del Este, podía moverse con una cierta tranquilidad gracias a su pasado comprometido en las filas del Partido Comunista Italiano y a su amabilidad connatural.

El proyecto de una gran edición crítica de las obras de Nietzsche todavía no existía, ni Montinari podía imaginar cuál sería la tarea que tendría que asumir:

Colli y él habían comprendido que había que reconstruir mucho, pero no imaginaban cuánto. Como Hans Castorp cuando llegó a la montaña mágica, Montinari pensaba quizás permanecer siete días en Weimar y, en cambio, se quedó siete años [en realidad cerca de nueve] solo para sentar las bases de la edición ${ }^{8}$.

Fue un compromiso de por vida: Montinari se mudó de manera estable a Weimar en 1965, y aquí se decidieron su vida privada y su destino como investigador. El «gran duque de Weimar», como lo definían los compañeros con afecto y deferencia, encontró desde el principio en aquel rincón de Turingia una patria intelectual y una amigable atmósfera9 .

6. F. Nietzsche, Aurora e Frammenti postumi (1879-1881), trad. de M. Montinari y F. Masini, Milano: Adelphi, 1964. En 1962 se había hecho el contrato con la editorial parisina Gallimard, mientras que para Alemania solo la sabia mediación de Karl Pestalozzi y la opinión autorizada de Karl Löwith convencieron a las ediciones De Gruyter a adquirir los derechos de Adelphi y Gallimard. La primera sección de la edición de Colli-Montinari en su lengua original (F. Nietzsche, Werke. Kritische Gesamtausgabe $=\mathrm{KGW}$ ), apareció en 1967 (Abt. 4, Bd. 1 y 2, que comprendía Richard Wagner in Bayreuth, Menschliches, Allzumenschliches I y II y los Fragmentos Póstumos del periodo). La edición crítica Colli-Montinari en lengua inglesa fue llevada a cabo por Stanford University Press.

7. Tutte le opere di Federico Nietzsche. Unica edizione italiana autorizzata in XI volumi, Milano: Monanni, 1926-1928.

8. C. Cases, «Il granduca di Weimar», en Mazzino Montinari. L'arte di leggere Nietzsche, cit., p. 12.

9. Un importante apoyo fue el de Helmut Holzhauer, de tradición marxista, desde 1945 director de las Nationale Forschungs- und Gedenkstätten der Klassischen deutschen Literatur de Weimar, de las que formaba parte también el Goethe-und-Schiller Archiv. «Anhelaba que la nueva edición de Nietzsche saliese de una editorial de la República Democrática Alemana y que se publicase paralelamente una historia política del Nietzsche-Archiv con la intención de poner las bases para una Nietzsche-Forschung marxista. En aquel periodo habíamos empleado muchos esfuerzos, demasiado inútiles, por llevar a cabo este proyecto. Cuando se puso de manifiesto su fracaso, también Helmut Holzhauer 
La complejidad del trabajo y las motivaciones que empujaron sobre todo a Montinari a implicarse en tal empresa se pueden reconstruir y comprender solo sumergiéndose en la fascinante correspondencia desde Weimar que Giuliano Campioni nos ha restituido ${ }^{10}$. Ya la primera carta a Colli de abril de 1961 comunica todo el fervor de aquellas «espléndidas jornadas de trabajo y de entusiasmo»:

Ante todo, alguna noticia personal. He encontrado aquí personas muy amables, no solo porque han puesto a mi disposición todo el material, que está conservado como sabes en el Goethe-Schiller-Archiv, sino también porque se han preocupado de buscarme un alojamiento mucho mejor del que tenía al principio. iVivo, de hecho, en la villa... de Nietzsche! Desde donde en este momento te escribo. Tengo para mí una habitación magnífica, con terraza y vista panorámica de Weimar por un lado y vista del jardín por donde Nietzsche enfermo había paseado. Aquí se respira un gran silencio.

La villa es de estilo «bayreuthiano»; pero situada como está en alto, y al estar un poco a las afueras de Weimar, es el lugar ideal para trabajar. He sentido una cierta emoción, muy mía y no comunicable a los demás, la primera vez que he cogido entre mis manos un m[anuscrito] de Nietzsche y luego cuando crucé el umbral de esta casa. No importa que haya desaparecido todo aquello a lo que hace referencia; el sitio mismo es sagrado. Créeme, desde que he comenzado a trabajar (el martes, porque el lunes es fiesta), casi sufro por la tensión y el deseo de concluir y porque veo que se necesita mucho tiempo todavía. Y lo mejor de todo es que lo hagamos todo de manera seria, nueva y definitiva [...] Este viaje es el más importante que he hecho en mi vida, quizás... Te estoy agradecido de haber tenido la idea de que viajase a Weimar, no lo he olvidado. iHaremos una gran edición-traducción de Nietzsche! ${ }^{11}$.

El legado nietzscheano del antiguo Nietzsche-Archiv de la Humboldtstraße, confiado al Goethe-und-Schiller-Archiv en 1951 por la administración militar soviética ${ }^{12}$, estaba más o menos ordenado, pero eran muchos los inéditos y, sobre todo, se planteaba todavía la espinosa cuestión del Nachlass. Los fragmentos póstumos, tres veces más numerosos que las obras editadas por el mismo Nietzsche, habían sido publicados a partir de la Grossoktavausgabe editada por el Archivo Nietzsche ${ }^{13}$ según un orden de carácter temático, cuya arbitrariedad debía aparecer a Montinari inmediatamente evidente, una vez que tuvo delante los numerosos cuadernos, rollos y folios sueltos. Montinari se dio cuenta desde el principio, incluso después de haber examinado algunos cuadernos de Nietzsche, de que no sería una tarea muy sencilla la corrección de los textos: se planteaba la alternati-

aceptó plenamente la forma ya definitiva de la edición nietzscheana, apoyándola con gran compromiso» (K.-H. Hahn, «Montinari a Weimar», en Mazzino Montinari. L'arte di leggere Nietzsche, cit., p. 51).

10. G. Campioni, Leggere Nietzsche, cit., p. 69.

11. Ibid., pp. 255-256.

12. Para la historia del fondo del Nietzsche-Archiv después de la Segunda Guerra Mundial, véanse D. M. Hoffmann, Zur Geschichte des Nietzsche-Archivs. Chronik, Studien, Dokumente, Berlin/ New York: de Gruyter, 1991, passim; P. D’Iorio, «Geschichte der Bibliothek und ihrer Verzeichnisse», en Nietzsches persönliche Bibliothek, ed. de G. Campioni, P. D'Iorio, M. C. Fornari, F. Fronterotta y A. Orsucci, en colaboración con R. Müller-Buck, Berlin/New York: de Gruyter, 2003, pp. 33-77.

13. Gesammtausgabe (= GOA), ed. de A. Seidl, E. y A. Horneffer, H. Köselitz, E. Holzer, E. Förster-Nietzsche, Otto Weiß et al., Leipzig: Naumann, 1899-1904, en 15 vols. El volumen XV, publicado en 1901 con el prólogo de Elisabeth Förster-Nietzsche, contenía Der Wille zur Macht. Versuch einer Umwerthung aller Werthe (Studien und Fragmente). 
va entre renunciar a la proyectada edición italiana y comenzar ex novo. Sabemos cuál fue su decisión:

Por mi parte [...] te confirmo que debemos ver en la edición la tarea más importante, en un cierto sentido la única de mi vida en los próximos tres años. A eso dedicaré todas mis mejores energías. Desgraciadamente, no soy ahora como quisiera ser: es decir más incisivo, más fuerte, más fecundo en el trabajo, que es tan bello y me puede dar tanto — de esto estoy seguro- cuanto más me sumerjo en él. La «tristeza» está en el fondo - y nos proponemos que no se dé ${ }^{14}$.

Los problemas relacionados con el estado de los textos de Nietzsche fueron dados a conocer a los estudiosos internacionales en julio de 1964, en el curso del séptimo Colloque philosophique international de Royaumont: mientras que Colli era más bien reticente a participar, Montinari pensó en el congreso como en una batalla en la que combatir y prepara, desde el despacho, los planes de guerra ${ }^{15}$. Están en juego no solamente la búsqueda de un editor alemán, sino sobre todo la comprobada falta de fiabilidad de la Grossoktavausgabe, y la consiguiente necesidad de una nueva edición crítica, que había que defender todavía.

El congreso, que tuvo lugar en la abadía de Royaumont organizado por un joven Gilles Deleuze, y estuvo presidido por el eminente Martial Guéroult —según Alan Schrift «el primero en el que Nietzsche fue tratado por primera vez en Francia seriamente como filósofo» y que jugó un papel significativo en la legitimación de su reputación filosófica ${ }^{16}$ - contaba con la participación de conocidos académicos como Jean Wahl, Jean Beaufret, Karl Löwith, Eugen Fink, pero también de filósofos más jóvenes (Michel Foucault, Gianni Vattimo) y literatos de vanguardia (entre otros, Pierre Klossowski). Tenía razón Montinari en confiar en un público semejante, y por lo demás sus motivaciones eran estrictas:

El problema de una nueva edición de Nietzsche, hoy, no tiene solamente una importancia puramente filológica. En realidad, aunque existen numerosas ediciones de Nietzsche, se puede decir que la más importante, la Grossoktavausgabe, no puede ser considerada verdaderamente crítica y fiable. Es verdad que hay que reconocer grandes méritos a esta edición, por ejemplo haber resuelto los problemas de desciframiento, y haber dado a conocer una parte notable de los fragmentos póstumos. Sus defectos, sin embargo, son graves, tanto desde el punto de vista de la cantidad del material publicado como de la cualidad de la publicación, de manera que las interpretaciones filosóficas de Nietzsche, no pudiendo disponer de una sólida edición de base, se desarrollan con notables dificultades ${ }^{17}$.

14. Carta a Colli del 15.5.1963, en G. Campioni, Leggere Nietzsche, cit., p. 276.

15. Cf. G. Campioni, Leggere Nietzsche, cit., p. 131. Montinari escribirá después el 3 de marzo de 1967: «He recibido de París el volumen de los coloquios de Royaumont, lo he ojeado todo y me han venido a la mente muchas cosas. Nuestra intervención hace una figura óptima y nuestro proyecto es citado tanto en la introducción como en las conclusiones del inefable Gilles [Deleuze]. He pensado cómo, juntos, hemos combatido bien nuestra batalla y en cómo aquella decisión nuestra fue decisiva para los destinos de la edición» (ibid., p. 403).

16. A. D. Schrift, French Nietzscheanism, en Íd. (ed.), Poststructuralism and Critical Theory's Second Generation, NY: Routledge, 2014, pp. 19-46, cita p. 26.

17. G. Colli y M. Montinari, «État des textes de Nietzsche», en Nietzsche, Cahiers de Royaumont, Paris: Minuit, 1967, pp. 127-140. Edición italiana más detallada: «Stato dei testi di Nietzsche»: Il Verri 39/40 (1972), pp. 58-68, cita p. 58. 
Una prueba concluyente, en particular, es el modo en que se publicarán los fragmentos póstumos, es decir, el material de los cuadernos, considerándose independientes en cierta medida de las obras publicadas («ellos nos dan realmente algo que no se encuentra en las obras»). La distribución sistemática seguida hasta ahora por los editores de la Grossoktavausgabe, además de ser ilegítima, no permite trazar un hilo coherente del desarrollo de las ideas de Nietzsche, ni reconstruir las fases de gestación de los escritos editados. Peor todavía es el caso de los volúmenes que se refieren a la «parte más problemática de la obra de Nietzsche», la llamada Voluntad de poder: aquí el orden arbitrario consiente, por ejemplo, que se pongan juntos fragmentos que son de distintas épocas, con consecuencias, por lo menos, engañosas.

Una de las mayores decisiones que Montinari tomó en sus estancias en Weimar, destinada a cambiar el estatus de los textos y el enfoque dado a la interpretación, es la siguiente:

Es oportuno considerar los fragmentos póstumos según la perspectiva en que el mismo Nietzsche los consideraba, es decir, como apuntes, perfiles, esbozos con vista a las futuras obras. Pero, en este sentido, es necesario que tales fragmentos sean presentados tal como han sido escritos: se necesita, por tanto, que nosotros publiquemos todos los cuadernos de apuntes escritos por Nietzsche, siguiendo el orden cronológico de su redacción ${ }^{18}$.

Montinari apoya sus afirmaciones con numerosos ejemplos, que demuestran cómo «el estado de los textos de Nietzsche» es verdaderamente deplorable: cuadernos significativos que habían pasado casi inadvertidos (hoy es conocido el caso del M III 1, al que Nietzsche confió sus más completas consideraciones sobre el eterno retorno); textos en condiciones de abrir perspectivas nuevas (como los manuscritos que se remontan a la redacción de la cuarta Intempestiva, que contienen la verdadera toma de posición de Nietzsche frente a Wagner, más allá de la condescendencia de la obra editada); errores de desciframiento y omisiones ${ }^{19}$.

Por lo que respecta a la tan discutida obra La Voluntad de poder, tal obra, simplemente, «no existe», como resultará claro a todo estudioso que quiera apoyarse directamente en el material del $\operatorname{archivo}^{20}$.

18. «Stato dei testi di Nietzsche», cit., pp. 58-59. Esta decisión emergió precozmente, desde la segunda estancia. «En cuanto a la edición, cuando vuelva debemos hablar mucho de todos los problemas que comporta. Ahora tengo una idea mucho más precisa y al final de este periodo habré aclarado muchas cosas. Sin embargo, me parece que una cosa es cierta: los manuscritos van descifrados y transcritos por completo, estudiados como grupo, como manuscrito individual, como una página (ien muchos casos!), por lo tanto ordenados cronológicamente [...]. Una interpretación a fondo con lectura y transcripción de todo podría dar resultados filológicos todavía más precisos. Si esto es importante para los póstumos de una obra publicada por Nietzsche lo es mucho más para el conjunto de manuscritos no utilizados. Es decir, la lectura y transcripción de todo pone ante nosotros la elaboración de un pensamiento desde una libreta a un cuaderno, desde este cuaderno a otro, y así se obtiene con criterios internos la cronología o, mejor, la sucesión. iTodo esto no se había hecho hasta ahora!» (carta a Colli del 21.8.1961, G. Campioni, Leggere Nietzsche, cit., pp. 263-264).

19. "Como se deduce de la intervención 'État des textes de Nietzsche', la importancia de la nueva edición no resulta de un plan apriorístico ni abstracto de publicación, ni de una discusión sustancial sobre la mayor o menor importancia del Nachlass, sino de los resultados concretos...» (G. Campioni, «Mazzino Montinari», Dizionario Biografico, cit.).

20. Cf. M. Montinari, «La volonté de puissance» n'existe pas, ed. de P. D’Iorio, Paris: Éditions de l'éclat, 1996. 
Solo el minucioso trabajo, la paciencia y la pericia permitieron a Montinari deshacer este mito. Su primer acercamiento a los cuadernos, de hecho, parece mantenerse todavía en el cauce de la tradición, tal y como revela el léxico («aforismos» en vez de «fragmentos»):

En la segunda fase [del trabajo] que está todavía en curso, he pasado a la «Voluntad de poder». En estos días estoy estudiando cuatro manuscritos que forman el único núcleo ordenado por el mismo Nietzsche pero sin el título Wille zur Macht. Tres de esos manuscritos contienen 372 aforismos numerados y el cuarto contiene un índice a los tres manuscritos. ¿̇or qué he comenzado por aquí? Porque sostengo que este, siendo el único grupo de aforismos numerados, debe constituir el núcleo tanto de la traducción como de la edición ${ }^{21}$.

El trabajo será más largo y complejo de lo previsto. Ya al año siguiente, el desciframiento y el trabajo de copiar a máquina los manuscritos W II 1-4 inducen a Montinari a un estudio más profundo, que insinúa muy pronto la duda sobre la colocación y sobre el papel de tales manuscritos de síntesis. Montinari dice que «estaba sumido en un cierto proceso interior $»^{22}$, probablemente la señal de que se encontraba cerca de una intuición fundamental respecto a la Voluntad de poder:

Esta colección de fragmentos [de 1888], una vez terminada, la llamó el mismo Nietzsche: «la primera redacción de mi "proyecto de una transvaloración”»; él nunca pensó publicarla bajo aquella forma [...]. Sin embargo, Nietzsche consideró que esta primera redacción estaba terminada, y esa sigue siendo también la única recopilación — de algún modo ordenada y unitaria - de los apuntes para La voluntad de poder ${ }^{23}$.

He avanzado en el trabajo en esta semana prefestiva. En el archivo: lunes, en lugar de continuar la transcripción de W II 3, he trabajado al mismo tiempo en el Dm [Druckmanuskript $=$ manuscrito para la imprenta] de GD y AC así como en las carpetas que hacen referencia a estas dos obras, obteniendo resultados notables sobre la cronología y sobre la génesis de GD y AC. Me parece interesante que el material de GD y de una parte relevante de AC se encontrase, en agosto de 1888 , reunido en una Reinschrift [copia en limpio] que Nietzsche había escrito para WzM; a finales de agosto Nietzsche decidió concentrar el material sobre el cristianismo en una obra aparte ( $\mathrm{AC}=\mathrm{I}$ libro de la Umwerthung) y anticipar en GD su «filosofía in nuce» (junto a otros capítulos de carácter literario que difícilmente habrían encontrado un lugar en la Umwerthung). No es una novedad, pero el hecho de que nos pueda indicar todos los particulares de sus manuscritos me parece importante; así se puede fijar también la cronología de los manuscritos y se puede dar un sentido a los diversos planes del período ${ }^{24}$.

El conspicuo material para la proyectada obra, y en particular los apuntes de la primavera de 1888, muestran cómo Nietzsche se afana en llevar a cabo un signifi-

21. Carta a Colli del 21.8.1961, G. Campioni, Leggere Nietzsche, cit., p. 261.

22. «Busco captar bien y formular el significado del trabajo que estoy haciendo, si bien me han venido ideas para la introducción a los W II 1-4, pero no todavía una idea que me satisfaga y que responda a la pregunta: ‘¿Qué es lo que pretendía Nietzsche cuando tituló los 372 aforismos?’» (carta a Colli del 26.8.1962, G. Campioni, Leggere Nietzsche, cit., p. 269).

23. G. Colli y M. Montinari, «Notizie e note», en Opere VIII/2, p. 436.

24. Carta a Colli del 30.12.1964, G. Campioni, Leggere Nietzsche, cit., p. 344. 
cativo giro teorético y que, en realidad, sintiese que su objetivo se alejaba progresivamente. El abandono del «gran» proyecto y la fragmentación en la realización de una serie de escritos fue debido, según Montinari, no solo a la desconfianza de un proyecto sistemático que repugnaba a su naturaleza más profunda, sino también a un cierto vacío especulativo, después de que los conceptos basilares que habían servido de instrumentos para su investigación se le hubieran desmoronado en las manos a causa de su escepticismo ${ }^{25}$.

Para que la Voluntad de poder se convirtiese en una obra de Nietzsche tenía que intervenir el momento artístico, como se puede constatar de cada una de las obras editadas. Esto no sucede ni siquiera en su primer proyecto, y lo demuestra la numeración del mismo Nietzsche en estos cuadernos, que es una simple numeración material según el orden de las páginas, no una numeración «arquitectónica». Arquitectónica es solamente la distribución parcial del material escogido en los cuatro libros en los que entonces quería dividir la obra. No había nada, por lo tanto, del Nietzsche artista. Sin embargo, como es sabido, se quiere presentar igualmente la Voluntad de poder "como si» hubiese sido producida asimismo artísticamente por Nietzsche, o sea, si manipuló, si mutiló, si desmembró, si aumentó, si sistematizó aquel material con la presunción de poderse sustituir, de tener la vocación, el destino y la autorización de sustituirse por Nietzsche artista. El producto fue engañoso, no en el sentido en el que Nietzsche entiende el término, sino engañoso como puede serlo el resultado de una falsificación utilitaria ${ }^{26}$.

Con la edición de Colli-Montinari, el debate en torno a la Voluntad de poder podía darse por definitivamente cerrado.

Nace y se define así esta grandiosa empresa, entre las mayores de la posguerra, que restituyó a Nietzsche en la cultura depurado de escorias ideológicas y operaciones abusivas. Durante años de trabajo se fueron sacando a la luz valiosos inéditos y crecieron en más de 1500 páginas los fragmentos póstumos ${ }^{27}$; se corrigieron errores de desciframiento, de traducción y de datación; fueron desmontadas las recopilaciones arbitrarias. Pero, sobre todo, comenzaron a ver la luz los aparatos críticos, que pudieron reconstruir, en torno a las obras, los póstumos y las cartas, aquel contexto y aquellos lazos infra y extratextuales imprescindibles para una correcta comprensión e interpretación ${ }^{28}$.

Montinari respondía de la siguiente manera al germanista Paolo Chiarini, al que invitó en 1970 a dar un curso en la Universidad de Roma:

25. Cf. G. Colli y M. Montinari, «Notizie e note», en Opere VIII/3, p. 422.

26. G. Colli y M. Montinari, «Notizie e note», en Opere VIII/2, p. 422.

27. La edición no tiene, sin embargo, solo virtudes. Un defecto se refiere a la difícil distinción entre fragmentos y redacciones preparatorias, como ha subrayado W. Groddeck, "Vorstufe" und 'Fragment'. Zur Problematik einer traditionellen Unterscheidung in der Nietzsche-Philologie», en M. Stern (ed.), Textkonstitution bei mündlicher und schriftlicher Überlieferung, Tübingen: Niemeyer, 1991, pp. 165-175.

28. En este sentido fue importante la publicación, en los años ochenta, de la edición de bolsillo (Sämtliche Werke. Kritische Studienausgabe in 15 Bänden, München/Berlin: DTV/de Gruyter, 1980, ${ }^{3} 2009$ [= KSA]; Sämtliche Briefe. Kritische Studienausgabe in 8 Bänden, München/Berlin: DTV/de Gruyter, 1986, 22003 [= KSB]), que además de reproducir cronológicamente todos los fragmentos póstumos desde 1869 a 1889 anticipaba, en los volúmenes 14 y 15, los resultados del comentario y del aparato crítico in fieri. KSB publica solo las cartas de Nietzsche pero no tiene aparato crítico. 
Para el contenido del curso preferiría proceder por vía inductiva, comenzar por los problemas concretos de la crítica del texto de Nietzsche, en primer lugar, de las obras publicadas por él mismo, luego de las que dejó listas para la publicación (como Ecce homo, que es naturalmente el ejemplo más clamoroso), finalmente los problemas de la publicación del Nachlaß (y aquí la «voluntad de poder» representa el problema más vistoso). Naturalmente, se deberán tratar todas las cuestiones de exégesis y comentario: lecturas de Nietzsche en primer lugar, relación biografíaobra, el ambiente histórico-cultural. Sin embargo, pienso que el acento ha de ponerse sobre la lectura crítica de Nietzsche, cuyo componente «filológico» ha sido abandonado casi totalmente, a pesar de las ruidosas discusiones ${ }^{29}$.

Montinari no fue solo, de hecho, un filólogo y editor. Su tarea como intérprete se desarrolló paralelamente al esfuerzo editorial y marcó una novedad radical en el enfoque de Nietzsche, gracias a la precisión filológica, al planteamiento histórico y al uso de las fuentes ${ }^{30}$. Al trabajo sobre los manuscritos lo acompaña una reflexión constante, que puede ser seguida sobre todo en los cuadernos en los que anotaba pensamientos y reflexiones «que el ascetismo voluntario del historiador y del filólogo tiene, por un largo periodo, solo como presupuestos del trabajo» ${ }^{31}$. Las cartas dan testimonio de un cierto desasosiego respecto a la mera actividad archivista - por más que sea compleja y necesariamente interpretativa: la necesidad es la de conocer todo, de absorber todo, llegar a los términos de Nietzsche para poseerlo, también para calmar los propios tormentos existenciales:

\begin{abstract}
A menudo, especialmente en estas dos semanas, me siento informe, gris, deshuesado, inerte; el único rastro de entusiasmo me llega del trabajo, de N., en particular Humano es el libro que me sentiría capaz de suscribir casi entero - quisiera que discutiéramos juntos, por ejemplo, sobre el «filosofar histórico» y sobre la «ciencia» como N. los ve en esta obra, que no es de hecho «historicista» ni «positivista», el objetivo del autor es demasiado amplio y profundo [...] Necesito un desarrollo continuo que tiene - entonces - como la primavera una especie de pasión rabiosa por la verdad. Este es el sentido que tiene para mí ocuparme de Nietzsche.
\end{abstract}

Para mí Nietzsche se está convirtiendo en una gran prueba, me siento llamado continuamente a tomar posición, y deseo ya llegar a comprenderlo, es decir, a hacerlo mío. No sé decirte cómo este trabajo llega a ser cada día más mío, porque es mi tormento. Desde este atormentarme con la riqueza y el esplendor, pero también la miseria, la desorientación, la enfermedad de todo Nietzsche, quiero alcanzar MI posición con respecto a él. Y te agradezco que hayas hecho posible que tenga este trabajo, que es nuestro trabajo ahora.

Y más tarde:

29. Citado en G. Campioni, Leggere Nietzsche, cit., pp. 99-100.

30. Su ensayo (Nietzsche) se publicó en Adelphi en 1967, en una serie de amplia difusión, y fue reimpreso en 1975 (Che cosa ha veramente detto Nietzsche, Roma: Ubaldini). Son numerosos sus artículos y ensayos en revistas especializadas y colecciones internacionales, además de traducciones y prólogos. Montinari fue además fundador, en 1970, de la revista Nietzsche-Studien, órgano importante internacional de la investigación nietzscheana.

31. G. Campioni, Leggere Nietzsche, cit., p. 71. 
Los días pasan y cada vez me voy sumergiendo más no solo en el aparato (sobre el cual doy referencia de noticias y problemas todavía en una próxima carta filológica), sino también en las ideas y en el mundo de Zaratustra. Tengo que decir que para mí el trabajo con Nietzsche es siempre lo que más fuerza me da e incluso el placer de vivir $^{32}$.

Frente a las perplejidades de Colli, que temía que una proyectada biografía de Nietzsche pudiese distraerlo del trabajo de la edición, que se iba complicando cada vez más en el momento en que comenzaban a salir los primeros volúmenes italianos y franceses, Montinari reivindica con fuerza la necesidad de contrarrestar «un público viciado de los lugares comunes historicistas», la voluntad de decir «algo importante», y esta vez no a través de recensiones genéricas o artículos sensacionalistas: «se podría inaugurar un nuevo modo de pensar a Nietzsche» ${ }^{33}$, en una dirección histórica y antimetafísica, filológica y no actualizante ${ }^{34}$.

Mazzino Montinari no era un nietzscheano. Por el contrario, era un estudioso [...] que nos ha puesto ante los ojos, utilizando un caso ejemplar, la rugosidad de la historia (según una expresión preferida de su maestro Delio Cantimori) y la circunspección, la dificultad, el método necesarios para alcanzar una correcta aproximación al pensamiento de un autor o a la reconstrucción de un particular momento histórico ${ }^{35}$.

Un método que después se ha convertido en escuela. En la Facultad de Lettere e Filosofia de la Università degli Studi de Pisa, en los primeros años de la década de los ochenta, un puñado de estudiantes, destinados a ser cada año más numeroso, se apasionaba con las lecciones de Giuliano Campioni ${ }^{36}$. El Nietzsche que Campioni, amigo y alumno de Montinari, abría ante sus ojos era bien distinto del pensador insano y fatal que se le había transmitido: escritor preciso, lector apasionado, estudioso informado, hombre de su tiempo y fino diagnosticador de la modernidad, en torno al cual tomaban cuerpo voces y rostros, fantasmagorías, esperanzas y desencantos de su siglo.

En nombre de Montinari, Campioni trasmitía a sus estudiantes —algunos de ellos hoy convertidos en investigadores - «antes de nada la convicción de que se razona a partir de hechos textuales, que los textos no son pretextos, que el rigor de la investigación filológica es un presupuesto indispensable para la fuerza del análisis» ${ }^{37}$. En segundo lugar, la referencia a la edición de Colli-Montinari como

32. Cartas a Colli del 22.8.1963, 11.11.1963 y 7.5.1967, G. Campioni, Leggere Nietzsche, cit., pp. 281, 292 y 417, respectivamente.

33. Cf. carta a Colli del 14.10.1965, G. Campioni, Leggere Nietzsche, cit., p. 377.

34. Esta posición hizo problemática la aceptación de la «Introducción editorial» a la edición de Adelphi de las Opere (1963) firmada a cuatro manos con Colli, que consideraba a Nietzsche en su «unidad estética» y antihistórica (cf. G. Campioni, Leggere Nietzsche, cit., pp. 82 ss.).

35. P. D’Iorio, Mazzino Montinari. L'arte di leggere Nietzsche, cit., p. 9.

36. En aquel tiempo Montinari enseñaba en la Universidad de Florencia. Se tendría que haber trasladado al poco tiempo a la Universidad de Pisa, pero la muerte prematura le sobrevino el día anterior del comienzo de curso, el 24 de noviembre de 1986. Después de su muerte, la edición ha sido llevada a cabo por Volker Gerhardt, Norbert Miller, Wolfgang Mueller-Lauter y Karl Pestalozzi para las Werke; por Norbert Miller y Annemarie Pieper para las Briefe.

37. Cf. la «Premessa» a Prospettive. Omaggio a Giuliano Campioni, ed. de M. C. Fornari, P. D’Iorio, L. Lupo y C. Piazzesi, Pisa: ETS, 2015, pp. XV ss. 
instrumento indispensable para aprender a leer a Nietzsche histórica y genéticamente; además, aquel ojo histórico que el mismo Nietzsche invocaba para ser buenos filósofos: el estudio de las fuentes, la reconstrucción del contexto. «Siguiendo a Campioni habíamos descubierto y profundizado toda aquella serie de referencias implícitas, de citas escondidas, de ilusiones paródicas que forman la trama del texto de Nietzsche y que contribuyen a dar sabor y espesor a su filosofía ${ }^{38}$. Desde este Nietzsche se destaca y, mejor dicho, se aprende a mirar con sospecha cada lectura e interpretación que tengan en el rigor histórico-filológico su indispensable premisa.

Gracias a la empresa de Colli y Montinari y al trabajo de Campioni y de sus discípulos, el valor de la escuela italiana en el ámbito de la Nietzsche-Forschung es hoy unánimemente reconocido: muchos investigadores y grupos de investigación de distintos países que comparten su metodología y objetivos, tanto en Europa como en América Latina, han dado vida a fructíferas colaboraciones y a proyectos comunes. La edición española de las Obras Completas, de los Fragmentos Póstumos y de la Correspondencia de Nietzsche, así como las numerosas iniciativas que han tomado los estudios de SEDEN (Sociedad Española de Estudios sobre F. Nietzsche), se sitúan en el cauce de esta apreciada herencia.

[Traducción del italiano: Luis E. de Santiago Guervós Universidad de Málaga]

38. Ibid., p. XVI. 